\title{
B-01
}

\section{DSS-SALTIRSOIL: UN SISTEMA DE AYUDA A LA DECISIÓN EN INTERNET PARA LA OBTENCIÓN DE RECOMENDACIONES DE GESTIÓN DEL RIEGO Y EL CULTIVO EN TIERRAS AMENAZADAS POR SALINIZACIÓN}

\author{
Visconti Reluy, F. (1) (P), de Paz Bécares, J.M. (2)
}

1 Titulado Superior, Instituto Valenciano de Investigaciones Agrarias, Centro para el Desarrollo de la Agricultura Sostenible, fernando.visconti@uv.es

${ }^{2}$ Colaborador Científico Adjunto, Instituto Valenciano de Investigaciones Agrarias, Centro para el Desarrollo de la Agricultura Sostenible, depaz jos@gva.es

\section{Resumen}

En el presente trabajo se presenta un ejemplo de utilización del sistema de ayuda a la decisión DSS-SALTIRSOIL para la recomendación del riego en una plantación de caqui "Rojo Brillante" en la zona de riegos del Magro de la Ribera del Xúquer (Valencia). De acuerdo con la simulación realizada con datos de la zona representativos de su meteorología (año 2010), suelo (franco arcilloso), calidad de agua (2.3 dS/m) y manejo del riego (goteo) y el cultivo, se esperaría una pérdida del $15 \%$ de producción como consecuencia de la salinización. Un incremento de la dotación anual de riego de $140 \mathrm{~mm}$, o una disminución de la salinidad hasta $2.0 \mathrm{dS} / \mathrm{m}$ reducirían dicha pérdidas a tan solo un $10 \%$ en dicho año de meteorología media. No obstante, la simulación del periodo 2000-2015 indica que una disminución de $100 \mathrm{~mm}$ en la precipitación anual media supone una amenaza de pérdida de producción de más del $20 \%$ en promedio. En consecuencia una solución satisfactoria a los problemas de salinidad en el cultivo del caqui en la zona pasa necesariamente por usar aguas de riego de salinidad más baja $(<2.0 \mathrm{dS} / \mathrm{m})$, o bien por un cambio del patrón del caqui a uno más tolerante a la salinidad como es el $D$. virginiana.

\begin{abstract}
In this article an example of utilization of the decision support system DSSSALTIRSOIL is shown for the recommendation of irrigation in a persimmon "Rojo Brillante" plantation in the Magro irrigation district in the Ribera del Xúquer (Valencia). According to the simulation carried out with data from the area, which is representative of the meteorology (2010), soil (clay loam), water quality ( $2.3 \mathrm{dS} / \mathrm{m}$ ) and irrigation (drip) and crop management, $15 \%$ yield losses would be expected as a result of salinization. An increase in the annual irrigation dose of $140 \mathrm{~mm}$, or a decrease in salinity down to $2.0 \mathrm{dS} / \mathrm{m}$ would reduce such losses to only $10 \%$ in such a year of average meteorology. However, the simulation of the span 2000-2015 indicates that an average decrease of $100 \mathrm{~mm}$ in the annual rainfall poses a threat of yield losses over $20 \%$ on average. Consequently a satisfactory solution for the salinity problems in persimmon cropping in the area necessarily involves using irrigation water of lower salinity $(<2.0 \mathrm{dS} / \mathrm{m})$, or replacing the persimmon rootstock by a more salttolerant one such as the $D$. virginiana.
\end{abstract}

\section{1- Introducción}


La amenaza de la salinización siempre está presente sobre el regadío, máxime cuando este se desarrolla en tierras con una o varias de las siguientes características: topografía deprimida, nivel freático elevado, agua de riego de calidad insuficiente, suelo de textura arcillosa y/o limosa, clima de árido a subhúmedo seco, y cultivo sensible a la salinidad. En España se estiman entre 0.6 y 1 Mha las tierras afectadas por salinidad principalmente bajo riego (FAO, 1997; Wicke et al., 2011). En la Comunidad Valenciana el $42 \%$ de los suelos de regadío presentan algún problema de salinidad con efectos limitantes sobre la producción (de Paz et al., 2004). En el escenario actual de creciente competencia por agua de calidad para usos urbanos, industriales y agrícolas, disponibilidad de aguas no convencionales, incremento de la aridez por el cambio climático, y expansión de cultivos muy sensibles como por ejemplo el caqui "Rojo Brillante", la salinización de suelos se perfila como un problema cuya importancia va a seguir aumentando en los próximos años.

Dejando a un lado la bioingeniería, los métodos con que tradicionalmente han contado los agricultores para superar los problemas de salinidad pueden clasificarse en tres tipos de mayor a menor efecto. En primer lugar para luchar contra la salinización se debe favorecer la infiltración, redistribución y drenaje del agua de riego y lluvia y en consecuencia, la lixiviación de las sales del suelo. Esto se consigue actuando sobre las propiedades hidrofísicas del suelo y el subsuelo mediante la instalación de sistemas de drenaje enterrado, cultivando en mesetas y favoreciendo la estructuración del suelo. Así se consigue aumentar la permeabilidad de los suelos y/o bajar el nivel freático. En segundo lugar, una vez aseguradas estas dos cosas, el agricultor cuenta con varias alternativas para luchar contra la salinización como son: i) aumentar la dosis de riego con una fracción de lavado que ayude a lixiviar las sales, ii) cambiar a aguas de mayor calidad, es decir, menos salinas, y/o menos cloruradas, y iii) cambiar a cultivos menos sensibles a la salinidad y/o el cloruro, o injertar en patrones más tolerantes. En tercer lugar y de manera complementaria el agricultor puede optimizar la fertilización, y/o estimular la tolerancia de los cultivos a la salinidad y/o el cloruro. Algunas de estas acciones es posible llevarlas a cabo y otras no, y a costes que se deben valorar para hallar la solución más sostenible.

La sencillez con que se puede actuar sobre la salinidad del suelo mediante el uso de una fracción de lavado ha hecho de este, un método muy popular de lucha contra la salinización. Así, desde mediados del siglo pasado se han propuesto métodos cada vez más sofisticados para calcular la fracción de lavado necesaria para bajar la salinidad del suelo hasta un nivel no perjudicial para el cultivo, esto es el cálculo del requerimiento de lavado (Visconti et al., 2012a). Por otro lado el regadío moderno no puede entenderse sin la programación de riegos que parte del cálculo de las necesidades hídricas de los cultivos, teniendo en cuenta además la eficiencia de los sistemas de riego y el aporte de las lluvias. Con la creación del sistema de ayuda a la decisión accesible en Internet DSS-SALTIRSOIL se ha logrado un sistema automatizado que programa los riegos mensualmente y recomienda otras prácticas de gestión agrícola considerando tanto las necesidades hídricas de las plantas como el requerimiento de lavado, y otras limitaciones y posibilidades de gestión del cultivo.

El objetivo de este trabajo es mostrar el funcionamiento del DSS-SALTIRSOIL mediante un ejemplo de gestión del riego, el suelo y el cultivo de caqui "Rojo Brillante" en el término municipal de Carlet (Valencia).

\section{2- Aplicación del sistema de recomendación DSS-SALTIRSOIL}

\subsection{Descripción del DSS-SALTIRSOIL}

El sistema de ayuda a la decisión DSS-SALTIRSOIL se encuentra alojado en el portal de Internet AGROSAL (http://agrosal.ivia.es/). Se trata de una web pensada para la 
difusión hacia el sector de contenidos relacionados con la salinidad de los suelos agrícolas en regadío (de Paz et al., 2015). El núcleo del DSS-SALTIRSOIL es el modelo SALTIRSOIL, diseñado para la predicción mensual de la salinidad en suelos de regadío con drenaje libre (Visconti et al., 2011; Visconti et al., 2014). Se trata de un modelo unidimensional de estado transitorio (transient-state), el cual simula el movimiento del agua en el suelo mediante un algoritmo capacitivo, y el movimiento de las sales mediante una aproximación de flujo pistón con dispersión hidrodinámica. El modelo SALTIRSOIL dispone, además, de un módulo llamado SALSOLCHEM que ajusta las concentraciones de las sales en la solución del suelo en virtud de su solubilidad, el contenido de agua, el contenido de carbonato de calcio y de yeso, así como el de $\mathrm{CO}_{2}$. Como resultado final de las simulaciones el modelo presenta al usuario la conductividad eléctrica a $25^{\circ} \mathrm{C}$ del extracto de saturación del suelo (CEe), así como su relación de adsorción de sodio (RASe), y su contenido de cloruro (Cle), y todo ello mes a mes durante el desarrollo del cultivo. El modelo SALTIRSOIL ha sido validado en varias parcelas ensayo en diferentes zonas de la Comunidad Valenciana y con diferentes cultivos (Visconti et al., 2010a; Visconti et al., 2012b; Visconti et al., 2014).

Los datos de entrada del modelo y en consecuencia del DSS-SALTIRSOIL se clasifican en cinco categorías. Por orden de importancia en la simulación de CEe estos son (Visconti et al., 2010b): meteorológicos (lluvia, evapotranspiración de referencia), calidad de agua de riego (contenidos de iones), cultivo (coeficientes de cultivo, marco de plantación, profundidad de enraizamiento), suelo (propiedades hidrofísicas, carbonato de calcio, yeso, $\mathrm{CO}_{2}$ ) y manejo del riego (dosis, frecuencia de riego y superficie humedecida). El DSSSALTIRSOIL se encuentra vinculado en el portal AGROSAL a una base de datos meteorológicos, de calidad de agua, cultivos y suelos georreferenciados y representativos de la Comunidad Valenciana. De este modo los agricultores y técnicos agrícolas que se enfrenten a problemas de salinidad, simplemente conociendo la ubicación de sus parcelas pueden realizar sus simulaciones, y obtener sus recomendaciones de gestión.

\subsection{El cultivo de caqui "Rojo Brillante"}

El caqui "Rojo Brillante" (Diospyros kaki L. cv "Rojo Brillante") es un cultivo arbóreo que presenta características agronómicas y comerciales muy interesantes como alternativa a cítricos y frutales de hueso en áreas de clima mediterráneo. En consecuencia su cultivo se encuentra en plena expansión, sobre todo en la Comunidad Valenciana, de donde con más de 220.000 t se obtiene más del $80 \%$ de la producción nacional de esta fruta (Arnandis, 2015). El caqui es, no obstante, un cultivo sensible a la salinidad, acusando una notable fitotoxicidad al cloruro sobre todo cuando se injerta sobre $D$. lotus, lo cual supone una de las limitaciones más importantes a que se enfrentan los agricultores (de Paz et al., 2016). La sensibilidad al cloruro es tal, que incluso en condiciones de ligera salinidad manifiesta síntomas adversos que incluyen menor calibre de los frutos cosechados, maduración acelerada y menor eficacia de los tratamientos poscosecha (Besada et al., 2016). Así pues, a continuación se mostrará cómo el DSS-SALTIRSOIL se puede aplicar para recomendar el manejo más idóneo de una plantación adulta de caqui "Rojo Brillante" en el término municipal de Carlet (Valencia). Carlet y su comarca, la Ribera Alta del Xúquer, constituye el centro de difusión pionero del caqui "Rojo Brillante" (Llácer et al., 2008), y donde a principios de la presente década se describió por primera vez en España la sintomatología que posteriormente se ha diagnosticado como fitotoxicidad al cloruro (de Paz et al., 2016).

\subsection{Simulación de la salinidad del suelo con DSS-SALTIRSOIL}

Desde la página web AGROSAL (http://agrosal.ivia.es/) (Fig. 1) pinchando sobre el botón DSS-SALTIRSOIL en el encabezado o bien pinchando en el logo abajo a la derecha accedemos al sistema. En esta primera página se presenta una descripción del DSSSALTIRSOIL, y mediante los botones de la columna a la izquierda podemos navegar hacia 
una guía de manejo, un vídeo demostración y el propio sistema, bien pinchando en "Recomendación de Riego" bien en su logo. Así accedemos a una página donde nos identificamos con nuestras credenciales de usuario. En caso de ser nuevos en el sistema, antes de poder utilizarlo nos registraremos.

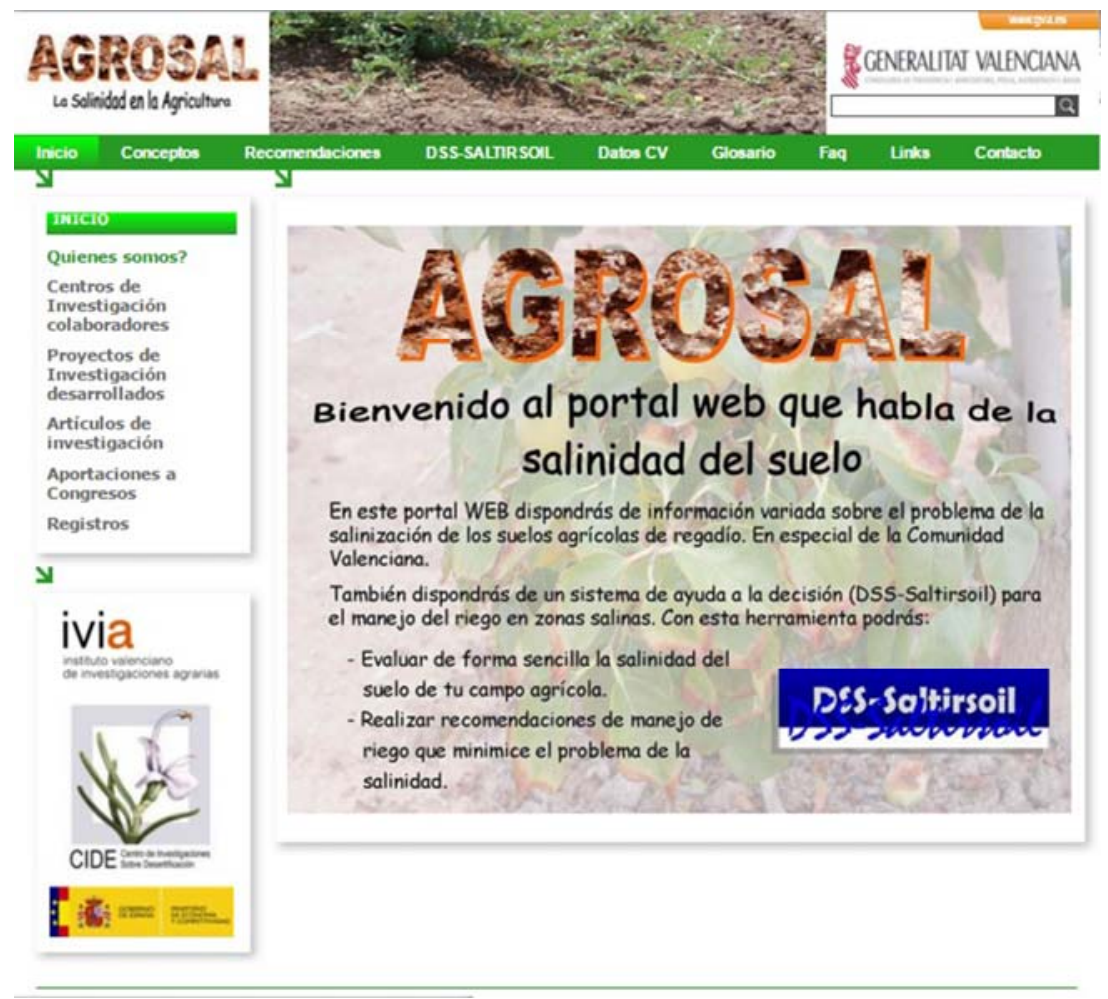

Figura 1. Entrada al portal AGROSAL.

Una vez dentro del sistema se nos presenta una página con diferentes pestañas mediante las cuales accederemos a los menús que nos permitirán seleccionar e introducir los datos necesarios para trabajar. En primer lugar en la pestaña «General» se nos pide un nombre para la simulación que queremos realizar. En nuestro caso escribimos por ejemplo «Caqui-Carlet», como provincia seleccionamos «Valencia», como término municipal «Carlet» y finalmente como profundidad para la simulación elegimos $60 \mathrm{~cm}$. Esta es una profundidad de enraizamiento razonable para un caqui adulto injertado sobre $D$. lotus, pie que se caracteriza por presentar una raíz muy fibrosa y en consecuencia, de desarrollo superficial. Para hacer efectivos estos datos en nuestra simulación pinchamos en el botón «Guardar», lo cual nos lleva a la siguiente pestaña.

En la pestaña «Clima» se presenta un cuadro de lista desplegable con los nombres de todas las estaciones meteorológicas que el «Sistema de Información Agroclimática para el Regadío» (http://eportal.magrama.gob.es/websiar/lnicio.aspx) tiene distribuidas en la Comunidad Valenciana. De entre estas estaciones, gestionadas por el Servicio de Tecnología del Riego (STR) del IVIA (http://riegos.ivia.es/), seleccionamos la estación de «Carlet CE Coop». Como año de simulación seleccionamos 2010, y a continuación se nos muestran los datos mensuales y el anual total de precipitación, número de días de lluvia, evapotranspiración de referencia y temperatura para dicha estación y año. El año 2010 ejemplifica con $518 \mathrm{~mm}$ de precipitación total, lo que es un año normal en una zona que se caracteriza por una precipitación media anual de $490 \pm 70 \mathrm{~mm}$ (IC95\%). Los datos que se presentan en este cuadro son editables pinchando en cada celda, cambiando el dato y haciendo clic en «intro». Así que en el caso de que el usuario disponga de datos propios, puede introducirlos y adaptar las simulaciones a la meteorología concreta de su parcela. 
Hacemos efectivos los cambios pinchando en el botón «Guardar», con lo cual pasamos a la siguiente pestaña.

En la pestaña «Cultivo» se presenta un cuadro de lista desplegable con los cultivos cuyos datos de coeficientes de desarrollo, tolerancia a la salinidad, etc., están incluidos en la base de datos del sistema. Seleccionamos «Kaki_Lotus», y dado que se trata de un cultivo multianual, como duración del cultivo seleccionamos 365 días, y como «Inicio cultivo» seleccionamos la fecha de 1 de enero de 2010. En caso de tratarse de un cultivo anual, p.ej., hortalizas, cereales, etc., seleccionaríamos la fecha de siembra o trasplante como «Inicio cultivo», y la fecha de cosecha como «Fin cultivo». A continuación en el cuadro «Riego» a la derecha, en el primer cuadro de lista desplegable seleccionamos de entre los tipos de riego «manta», «surcos» y «goteo», éste último. Con esta acción se habilitan varios cuadros que nos van a permitir introducir la información que caracteriza el riego por goteo de nuestra parcela como «Dosis mín. de cada riego» donde escribiremos $5 \mathrm{~mm}$, «No máx. riegos/mes» donde pondremos 20, «Diámetro de copa» donde introduciremos $3.8 \mathrm{~m}, \mathrm{y}$ finalmente «Distancia entre plantas», y «Distancia entre líneas» que serán $3.5 \mathrm{~m}$ y $4 \mathrm{~m}$ respectivamente. Estos datos arrojan un área sombreada de $11 \mathrm{~m}^{2}$ por árbol, lo cual supone un $79 \%$ de la superficie de la parcela. Finalmente introducimos los datos de «Porcentaje de suelo mojado» por el sistema de riego, que dejamos como 15\%, y « $\mathrm{N}^{\circ}$ goteros por árbol» y «Caudal del gotero» que serán en este ejemplo 4 y $4 \mathrm{~L} / \mathrm{h}$ respectivamente. Finalmente pinchamos en «Calcular Riego» y se nos muestra un cuadro con la programación mensual del riego adecuada para atender las necesidades hídricas de un cultivo de caqui con las características consideradas previamente. Vemos que en total se recomendarían $276 \mathrm{~mm}$, iniciándose los riegos en mayo con $43 \mathrm{~mm}$, y finalizándose en agosto con $83 \mathrm{~mm}$, y con un máximo de $87 \mathrm{~mm}$ en julio. Es importante destacar que estos datos también son editables, de tal modo que el usuario del sistema puede introducir los datos de las cantidades reales de agua que se utilizaron para regar su plantación. Una vez tenemos todos los datos introducidos de manera adecuada los hacemos efectivos pinchando en «Guardar» y pasamos así a la pestaña siguiente.

En la pestaña «Suelo» se presenta un cuadro de lista desplegable con suelos presentes en el término municipal donde tenemos la plantación, en este caso Carlet. Los suelos se encuentran clasificados en virtud de su textura y acompañando ésta, una estimación de la permeabilidad de los mismos. Elegimos un suelo franco arcilloso de permeabilidad media, cuyas propiedades se nos muestran por profundidades. Estos datos de suelo no son editables para asegurar su consistencia edafológica. Pinchamos en «Guardar» y pasamos a la siguiente pestaña.

En la pestaña «Agua Riego» se presentan varios cuadros de lista desplegable donde en orden de arriba abajo seleccionamos el término municipal, la unidad de demanda agraria y el origen del agua dentro de la misma. En nuestro caso seleccionamos respectivamente, «Carlet», «Riegos del Magro» y «Subterránea». En último lugar seleccionamos la salinidad del agua de riego, en este caso un valor de $2.3 \mathrm{dS} / \mathrm{m}$, es representativo del riego con agua subterránea, ya sea de pozo o manantial, en la zona de riegos del río Magro. En un cuadro al lado se nos muestran los datos de concentración de iones y otros parámetros de calidad del agua. Dada su salinidad, cloruro de $6.7 \mathrm{meq} / \mathrm{L}$, RAS de $2.1(\mathrm{mmol} / \mathrm{L})^{1 / 2}$ y carbonato de sodio residual (CSR) de $-13 \mathrm{meq} / \mathrm{L}$ el agua que hemos elegido se clasificaría como ligeramente salina, moderadamente clorurada, no sódica, y no alcalina (Fig. 2). Los datos del agua de riego no son editables para asegurar, del mismo modo que con los datos de suelo, su coherencia interna. Una vez seleccionados los datos de calidad de agua ya se puede realizar la simulación de la salinidad pinchando en el botón «Evaluar y Recomendar», lo cual nos lleva a la siguiente pestaña. 


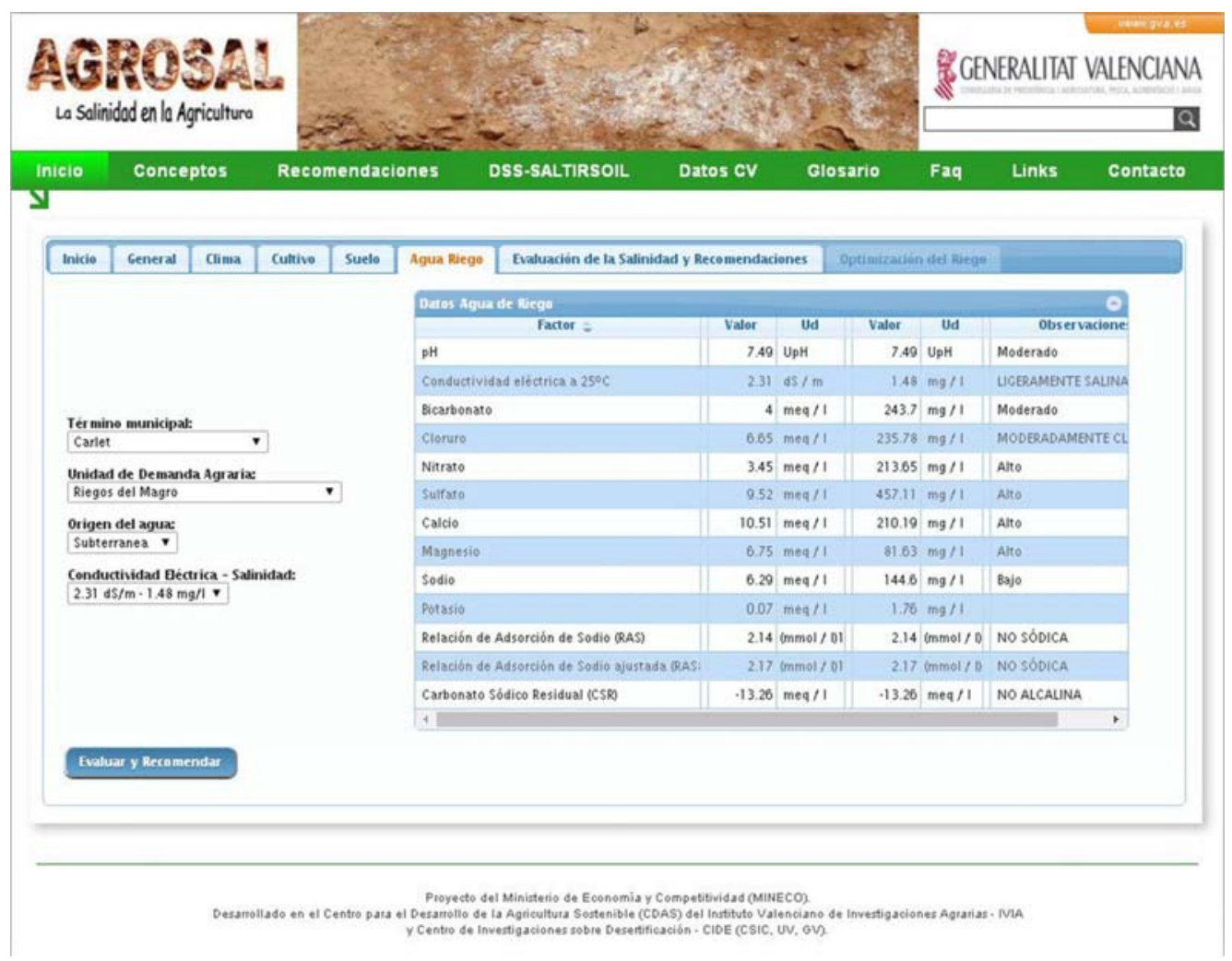

Figura 2. Pestaña «Agua Riego» con el agua seleccionada para la simulación.

En la pestaña «Evaluación de la Salinidad y Recomendaciones» se presentan los resultados de la simulación en forma gráfica, numérica y con mensajes en pantalla. En primer lugar llama nuestra atención la evaluación de ligera salinidad de la parcela, la cual ocasiona un descenso de la producción del 15\%. En las gráficas se presenta la evolución de CEe, RASe y Cle a lo largo del año (Fig. 3). Vemos así que la CEe varía entre 1.6 y 2.5 $\mathrm{dS} / \mathrm{m}$ a lo largo de la temporada de riegos (abril-noviembre), lo cual explica el problema de salinidad; que la RASe varía entre 0.9 y 2.8 (mmol/L) $)^{1 / 2}$, lo cual no ocasiona ningún problema de sodicidad; y que Cle varía entre 4.6 y $9.4 \mathrm{meq} / \mathrm{L}$, dando así lugar a una evaluación de ligera toxicidad al cloruro. Realizando la simulación para todos los años desde 2000 hasta 2015 se observa que la salinidad ha presentado una tendencia ascendente durante estos primeros años del siglo XXI (Fig. 4). Este hecho contribuye a explicar que el problema de fitotoxicidad al cloruro no se manifestase con claridad hasta comienzos de la presente década. Los mensajes de recomendación en pantalla nos indican las alternativas principales de que disponemos para mejorar el manejo del cultivo y evitar así los problemas de salinidad: «Optimizar el riego para lavar sales del suelo», «Cambiar a un agua de riego de salinidad más baja», y «Cambiar a otro cultivo o patrón más tolerante a la salinidad», y también para evitar los problemas de toxicidad al cloruro mediante mensajes similares. En el caso de la sodicidad se presenta un escueto «El manejo del riego es adecuado para evitar problemas de sodicidad». Todos los datos seleccionados para la simulación así como los resultados pueden descargarse como documento «pdf» pinchando para ello en el botón «Informe PDF». Dados los problemas descubiertos mediante la simulación, a continuación calculamos la programación de riegos que permitiría atender las necesidades hídricas del cultivo a la vez que se lavan sales para evitar el problema de salinidad que ocasiona la pérdida de producción del 15\%. Así pinchamos en el botón «Optimizar Riego» lo cual nos lleva a la siguiente pestaña. 


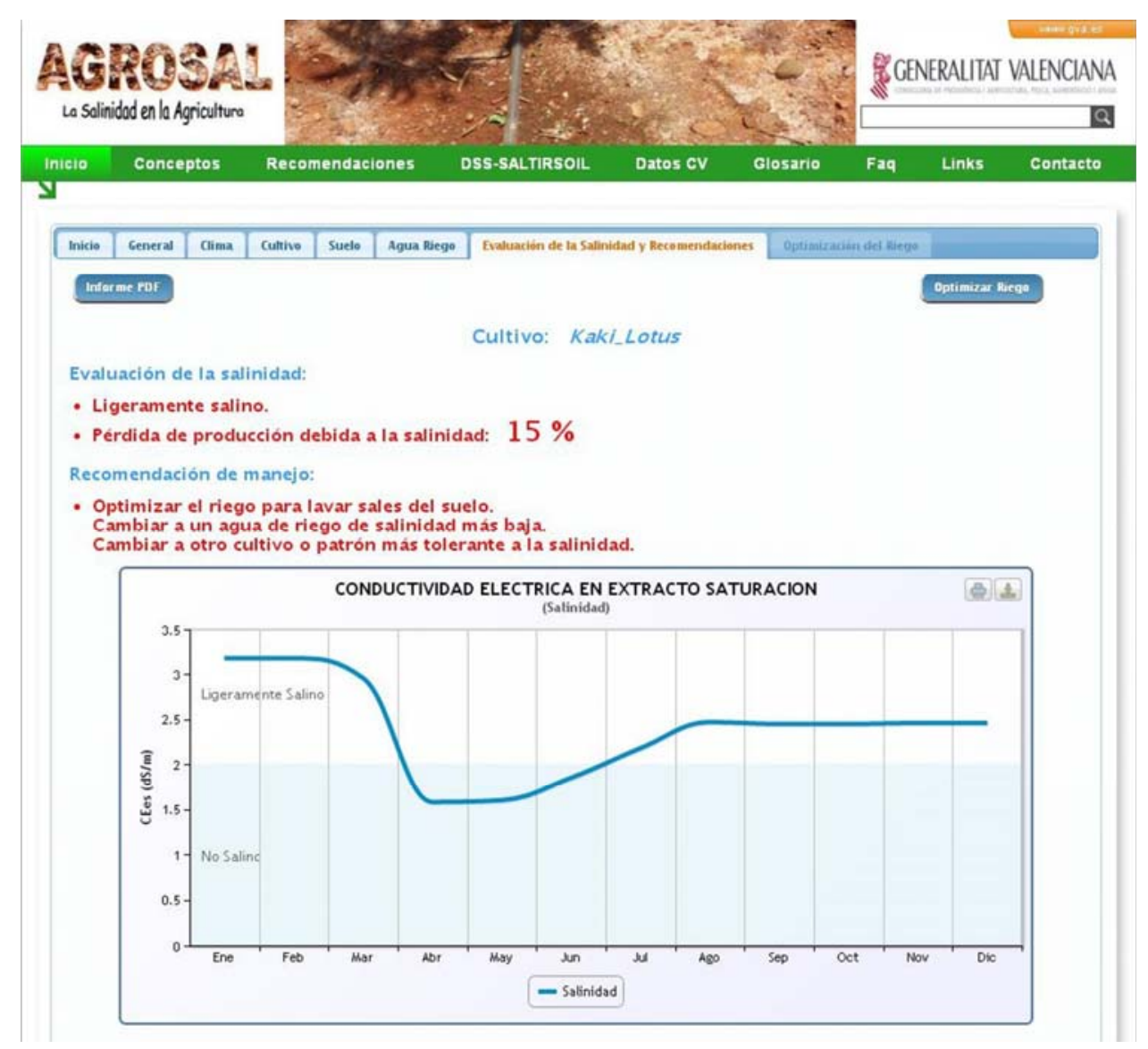

Figura 3. Pestaña «Evaluación de la Salinidad y Recomendaciones».

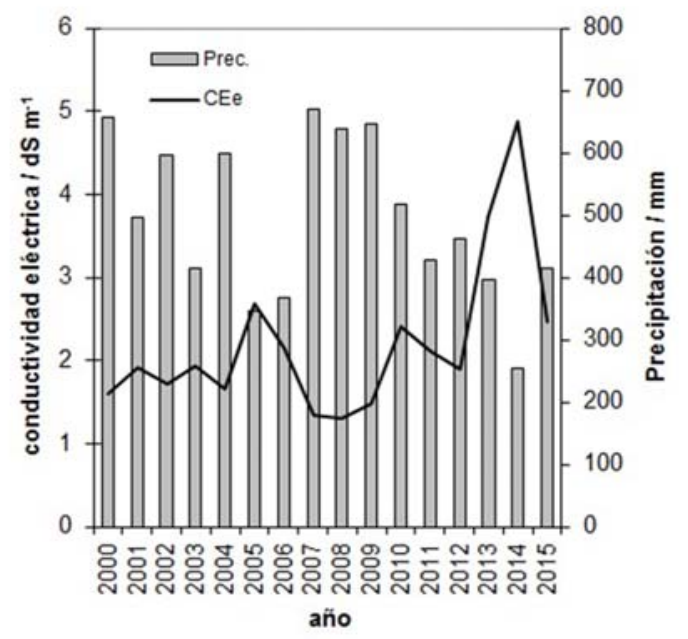

Figura 4. Evolución de la salinidad y precipitación promedio desde 2000 hasta 2015.

\subsection{Optimización de la gestión del riego, el suelo y el cultivo}

En la pestaña «Optimización del Riego» el sistema muestra los resultados de los cálculos de optimización. Estos son en primer lugar una programación mensual de riegos capaz de disminuir la pérdida de producción a tan solo un $10 \%$. El riego optimizado de este modo asciende ahora a $417 \mathrm{~mm}$ anuales, $140 \mathrm{~mm}$ más que el calculado teniendo únicamente en cuenta las necesidades hídricas del cultivo. En segundo lugar se presentan 
también en pantalla las gráficas con la evolución temporal tanto de la CEe, como de la RASe y el Cle. En este caso la CEe presenta valores entre 1.1 y $2.2 \mathrm{dS} / \mathrm{m}$ durante toda la temporada de riegos, mientras que el Cle se mantiene entre 3.3 y $8.1 \mathrm{meq} / \mathrm{L}$, estos son valores inferiores en más de $1 \mathrm{meq} / \mathrm{L}$ a los anteriores. Igual que antes todos estos resultados pueden descargarse como «pdf» pinchando en «Informe PDF».

Una mayor reducción de la salinidad, esto es, buscar una salinidad conducente a un $0 \%$ de pérdida de producción no se contempla en el sistema. Esto es debido a que no suele resultar una práctica sostenible buscar tales reducciones, dado que no existe una relación lineal entre la salinidad del suelo y la fracción de lavado (Ayers y Westcot, 1985; Visconti et al., 2012a) y en consecuencia tampoco la dosis de riego. En consecuencia, a medida que disminuye la salinidad, para obtener un mismo porcentaje de reducción de la misma, se necesitan incrementos cada vez mayores de la dosis de riego. Otras limitaciones son la cantidad de agua de riego, p.ej. el sistema nunca recomendará, en general, dosis de más de $1200 \mathrm{~mm}$ anuales, ni en particular para los cítricos de más de $800 \mathrm{~mm}$ anuales. En caso de que el único camino para alcanzar rendimientos del 90\% fuese regar por encima de dichas cantidades anuales, se recomienda que el usuario opte por alguna de las otras recomendaciones relacionadas con la calidad del agua de riego o con el cultivo, esto es el cambio a un agua de mayor calidad, o bien a un cultivo o patrón más tolerante a la salinidad.

Ambas alternativas de manejo pueden también explorarse mediante el sistema DSSSALTIRSOIL. En primer lugar, manteniendo la programación de riegos tal y como estaba originalmente, tan solo se necesitaría cambiar a un agua de riego de $2.0 \mathrm{dS} / \mathrm{m}$ en lugar de $2.3 \mathrm{dS} / \mathrm{m}$, para hacer que la pérdida de producción fuese tan solo de un $10 \%$ en lugar del $15 \%$. En segundo lugar, el usuario puede obtener información acerca de qué otros cultivos arbóreos darían una producción de al menos el $90 \%$ en condiciones similares de manejo y en consecuencia, de salinidad. Estos serían el limonero, la higuera, el olivo, la palmera datilera, la vid para vinificación y para uva de mesa, el granado y el caqui injertado en $D$. virginiana.

EI D. virginana es otro caqui que también se utiliza como pie de "Rojo Brillante" en la Comunidad Valenciana, aunque de manera mucho menos extendida que $D$. lotus. Debe tenerse en cuenta en todo caso que esta estimación de los cultivos alternativos es un dato menos fiable que los anteriores proporcionados por el sistema y que deberemos realizar la simulación para confirmar que de verdad se trata de una alternativa. También es importante tener en cuenta que la estimación del rendimiento se lleva a cabo en términos relativos al potencial propio de cada especie y cultivar. Esto significa que, aunque el rendimiento en términos relativos del caqui "Rojo Brillante" sobre pie $D$. virginiana en las anteriores condiciones de salinidad sea superior que sobre $D$. lotus, en términos absolutos ocurre al revés dado el gran vigor que confiere el pie $D$. lotus a la variedad en comparación con $D$. virginiana.

\subsection{Incertidumbre en las simulaciones y optimizaciones a lo largo del tiempo}

Para una plantación dada, de todos los factores que influyen sobre la salinidad, sodicidad y toxicidad al cloruro y en consecuencia sobre las recomendaciones de manejo, el más variable es la meteorología, y en concreto la lluvia. Como se ha presentado mediante la Fig. 4, durante el periodo 2000-2015 la salinidad simulada por el sistema para la zona de Carlet ha aumentado siguiendo las fluctuaciones en la precipitación. Así que los años 2007, 2008 y 2009 que fueron, con 650 mm de precipitación media, muy húmedos para el clima de la zona, dieron como resultado una salinidad y cloruro en el suelo de $1.1 \mathrm{dS} / \mathrm{m}$ y $3.3 \mathrm{meq} / \mathrm{L}$ en promedio durante la temporada de riegos. Por el contrario los años 2013, 2014 y 2015 que se han caracterizado por ser, con tan solo $355 \mathrm{~mm}$ de precipitación media, entre secos y muy secos para el clima de la zona, dieron como resultado una salinidad y cloruro en el suelo de $3.5 \mathrm{dS} / \mathrm{m}$ y $14 \mathrm{meq} / \mathrm{L}$. En consecuencia para años muy húmedos como el trienio 
2007-2009, no se recomendaría ningún incremento de los riegos para lavar sales y cloruro. Por el contrario para aquellos años de secos a muy secos como el trienio 2013-2015 se recomendarían riegos que oscilarían entre 510 y $930 \mathrm{~mm}$ anuales. En las condiciones de la zona tales dotaciones pueden ser muy difíciles de satisfacer, sobre todo si superan los 800 $\mathrm{mm}$ como en 2014. En general, para un manejo medio del riego un descenso de la precipitación de $100 \mathrm{~mm}$ ocasionaría una pérdida de producción de más del 20\%. En consecuencia, para tratar de superar el problema de salinidad definitivamente sería más adecuado otro tipo de solución como buscar un agua de riego de menos salinidad y cloruro, específicamente de menos de $2 \mathrm{dS} / \mathrm{m}$, o la utilización del patrón $D$. virginiana.

\section{3- Conclusiones}

Como se ha mostrado mediante el ejemplo desarrollado en este trabajo, disponer de un sistema de ayuda a la decisión como es el DSS-SALTIRSOIL, resulta útil debido a que permite evaluar las diferentes posibilidades con que cuenta el agricultor para lograr rendimientos de cultivo aceptables en situaciones de riesgo de salinización del suelo. El sistema DSS-SALTIRSOIL presenta una notable flexibilidad de uso debido a su vinculación a bases de datos meteorológicos, de cultivo, suelo, calidad de agua, etc. y a las posibilidades que ofrece a sus usuarios de editar toda esta información para adaptarla en la medida de lo posible a las condiciones concretas de sus plantaciones.

\section{4- Recomendaciones}

En el ejemplo desarrollado en este trabajo se ha mostrado la utilidad del DSSSALTIRSOIL para estudiar las alternativas de manejo de riego y cultivo de una plantación de caqui "Rojo Brillante" en la zona de riegos del río Magro en la Ribera del Xúquer (Valencia). En esta zona y dadas sus condiciones climatológicas se deberían utilizar, en general, siempre aguas de menos de $2 \mathrm{dS} / \mathrm{m}$, y dotaciones de riego de entre 400 y $450 \mathrm{~mm}$ anuales para asegurarnos una producción no límitada por la salinidad. Únicamente si alguna de estas dos condiciones no puede cumplirse, se recomendaría el cambio de $D$. lotus a $D$. virginiana como patrón de la variedad "Rojo Brillante".

\section{5- Agradecimientos}

El portal AGROSAL fue desarrollado en el marco de los proyectos-14592-C02-01 y CGL2009-14592-C02-02 financiados por el Ministerio de Ciencia e Innovación. Fernando Visconti agradece así mismo las ayudas disfrutadas dentro del programa Val i+d financiado por la Generalitat Valenciana, y el programa "Juan de la Cierva" financiado por el Ministerio de Economía y Competitividad, para el desarrollo del DSS-SALTIRSOIL.

\section{6- Bibliografía}

Arnandis, C. (2015). Prólogo. En M. L. Badenes, D. S. Intrigliolo, A. Salvador \& A. Vicent (Eds.), El cultivo del caqui (pp. 11-13). Valencia: Generalitat Valenciana.

Ayers, R. S., \& Westcot, D. W. (1985). Water quality for agriculture. Irrig Drain Paper 29, Rev. 1. Rome: Food and Agriculture Organization of the United Nations.

Besada, C., Gil, R., Bonet, L., Quiñones, A., Intrigliolo, D., \& Salvador, A. (2016). Chloride stress triggers maturation and negatively affects the postharvest quality of 
persimmon fruit. involvement of calyx ethylene production. Plant Physiology and Biochemistry, 100, 105-112.

de Paz, J. M., Visconti, F., Tudela, L., Quiñones, A., Intrigliolo, D. S., Jordà, M. \& Bonet, L. (2016). La fitotoxicidad por cloruro en el cultivo del caqui: Descripción del problema. Agrícola Vergel: Fruticultura, Horticultura, Floricultura (en prensa).

de Paz, J. M., Visconti, F., Moreno, G., Molina, M. J., Ingelmo, F., Martínez, D. \& Sánchez, J. (2015). AGROSAL: Presentación de un nuevo portal web que trata sobre la salinidad de los suelos agrícolas de regadío. parte I. Agrícola Vergel: Fruticultura, Horticultura, Floricultura, 380, 37-42.

de Paz, J. M., Visconti, F., Zapata, R. \& Sánchez, J. (2004). Integration of two simple models in a geographical information system to evaluate salinization risk in irrigated land of the valencian community, spain. Soil Use and Management, 20, 333-342.

FAO. (1997). FAO/UNESCO Soil Map of the World, Revised Legend, with Corrections and Updates. World Soil Resources Report 60 (Reprinted with updates as Technical Paper 20 ed.). Wageningen: ISRIC.

Llácer, G., Martínez-Calvo, J., Naval, M. \& Badenes, M. L. (2008). From germplasm to fruit export: The case of 'rojo brillante' persimmon. Advances in Horticultural Science, 22, 281-285.

Visconti, F., de Paz, J. M., Molina, M. J., Ingelmo, F., Sánchez, J. \& Rubio, J. L. (2010a). Progress towards DSS-SALTIRSOIL: Monthly calculation of soil salinity, sodicity and alkalinity in irrigated well-drained lands. Proceedings of the Global Forum on Salinization and Climate Change, Burjassot (Valencia, España). pp. 37.

Visconti, F., de Paz, J. M., Martínez, D. \& Molina, M. J. (2014). Irrigation recommendation in a semi-arid drip-irrigated artichoke orchard using a one-dimensional monthly transient-state model. Agricultural Water Management, 138, 26-36.

Visconti, F., de Paz, J. M., Molina, M. J. \& Sánchez, J. (2012b). Advances in validating SALTIRSOIL at plot scale: First results. Journal of Environmental Management, 95, S31-S36.

Visconti, F., De Paz, J. M., Rubio, J. L., \& Sánchez, J. (2010b). Preliminary results for the global sensitivity analysis of SALTIRSOIL model outputs. Procedia - Social and Behavioral Sciences, 2, 7763-7764.

Visconti, F., de Paz, J. M., Rubio, J. L. \& Sánchez, J. (2011). SALTIRSOIL: A simulation model for the mid to long-term prediction of soil salinity in irrigated agriculture. Soil Use and Management, 27, 523-537.

Visconti, F., de Paz, J. M., Rubio, J. L. \& Sánchez, J. (2012a). Comparison of four steady-state models of increasing complexity for assessing the leaching requirement in agricultural salt-threatened soils. Spanish Journal of Agricultural Research, 10, 222-237.

Wicke, B., Smeets, E., Dornburg, V., Vashev, B., Gaiser, T., Turkenburg, W. \& Faaij, A. (2011). The global technical and economic potential of bioenergy from salt-affected soils. Energy and Environmental Science, 4, 2669-2681. 